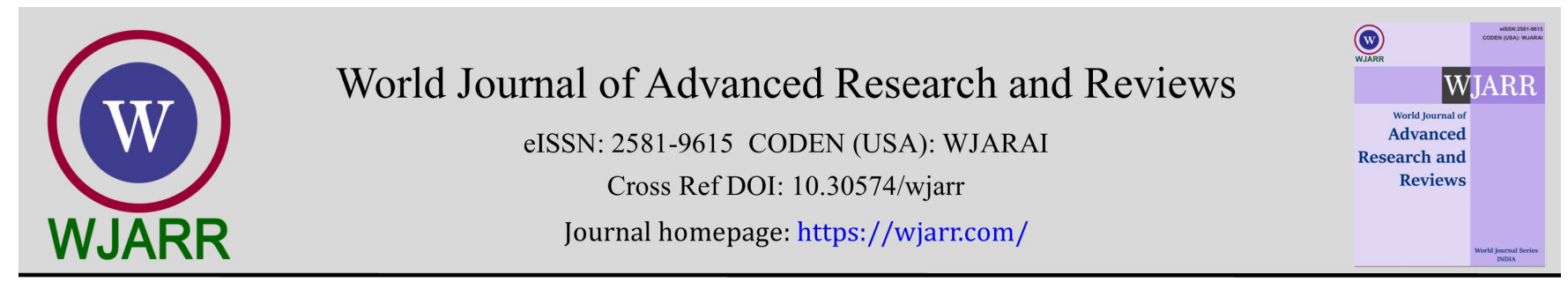

(RESEARCH ARTiClE)

\title{
Vehicle dynamics and tire models: An overview
}

Mohamed Belrzaeg 1, Abdussalam Ali Ahmed 1, ${ }^{*}$, Amhimmid Q Almabrouk 2, Mohamed Mohamed Khaleel 3, Alforjani Ali Ahmed ${ }^{4}$ and Meshaal Almukhtar ${ }^{5}$

${ }^{1}$ Mechanical and Industrial Engineering Department, Bani Waleed University, Bani Waleed/ Libya.

${ }^{2}$ Department of Mechatronics, Higher Institute of Engineering Technology Bani Walid/ Libya.

${ }^{3}$ Aeronautical Engineering Department, College of Civil Aviation-Misurata, Misurata/ Libya.

${ }^{4}$ College of Applied Sciences and Technology, Al-AWATA/ Libya.

${ }^{5}$ Mechanical Engineering Department, higher Institute of Engineering Technology, Bani Walid, Libya.

World Journal of Advanced Research and Reviews, 2021, 12(01), 331-348

Publication history: Received on 12 September 2021; revised on 16 October 2021; accepted on 18 October 2021

Article DOI: https://doi.org/10.30574/wjarr.2021.12.1.0524

\begin{abstract}
Stability control system plays a significant role in vehicle dynamics to improve the vehicle handling and achieve better stability performance. In order to study and evaluate the performance of the vehicles in addition to how to control it, it is necessary to identify obtain some models related to the dynamics of the vehicle as well as the tire models. This paper presents fundamentals of vehicle dynamics by introducing vehicle models and tire model, which have been widely adopted for vehicle motion control. This helps to get a basic idea of what parameters and states of a vehicle are important in vehicle motion control. This work is separated into four sections: vehicle planar model, full vehicle model, two degrees of freedom vehicle model (bicycle model) to design the controller, and wheel dynamic model.
\end{abstract}

Keywords: Vehicle dynamics; Vehicle model; Tire model; Stability; Planar model; Full model

\section{Introduction}

Vehicle Dynamics is an engineering discipline that deals with the motion of a vehicle in relation to its intended usage. It is a topic that is applied to a certain set of products, especially automobiles. Vehicle Dynamics employs theories and methods from mechanical engineering and machine design, as well as Control/Signal engineering and Human Behavioral Science [1].

In the 1990s, the global car industry and market structure underwent extraordinary transformation. Vehicle safety, environmental preservation, and intelligent control are all in high demand. As a result, innovative technologies like computer technology, virtual reality technology, and clever algorithms have become commonplace in the automobile business. Vehicle dynamics are crucial to the advancement of the automobile industry. Early vehicle dynamics research focused on different working conditions and service performance under external excitation. [2]. Researchers began to work on steering, suspension mechanics, and driving stability in the 1930s. The impacts of the external environment (such as road surface roughness, airflow, tire and driver) on vehicle dynamics, as well as the coupling interaction of these conditions, were examined by Lanchester Maurice and Segel. [3]. Segel [4] published a thorough review of vehicle dynamics achievements prior to 1990 in the Proceedings of the Institution of Mechanical Engineers in 1993. Vehicle ride comfort and handling stability research has gotten a lot of attention in the subsequent decades. The lateral or transverse dynamics of the vehicle are dealt with in handling dynamics, which primarily apply to vehicle handling stability, vehicle sideslip induced by tire lateral force, yawing, and roll motion.

\footnotetext{
* Corresponding author: Abdussalam Ali Ahmed

Mechanical and Industrial Engineering Department, Bani Waleed University, Bani Waleed/ Libya. 
The research on handling stability in vehicle dynamics progressed from experimental studies to theoretical analysis, from open loop to closed-loop. The representative monographs of vehicle handling dynamics include (Vehicle Handling Dynamics Theory and Application) by Abe M [5].

"Vehicle Handling Dynamics Theory" written by Guo [6]. Driving, braking, and ride comfort are all part of the vehicle's driving dynamics, which are separated into longitudinal and vertical dynamics. The research of vehicle longitudinal tire force solves the problem of driving and braking slip, while also improving driving and braking efficiency. The vertical tire force causes vehicle vibration and pitch movement, which affects ride comfort. Rajamani's monograph "Vehicle Dynamics and Control" is an example of a representative monograph. [7], Vehicle Dynamics Theory and Applications written by Zhang [8]. Furthermore, the longitudinal force of a tire when a vehicle is speeding up or stopping, as well as vehicle vibration induced by a working engine, are all part of the topic of vehicle dynamics research.

\subsection{Coordinate system}

The system of coordinates that is used to describe the vehicle motion as shown below in the figure

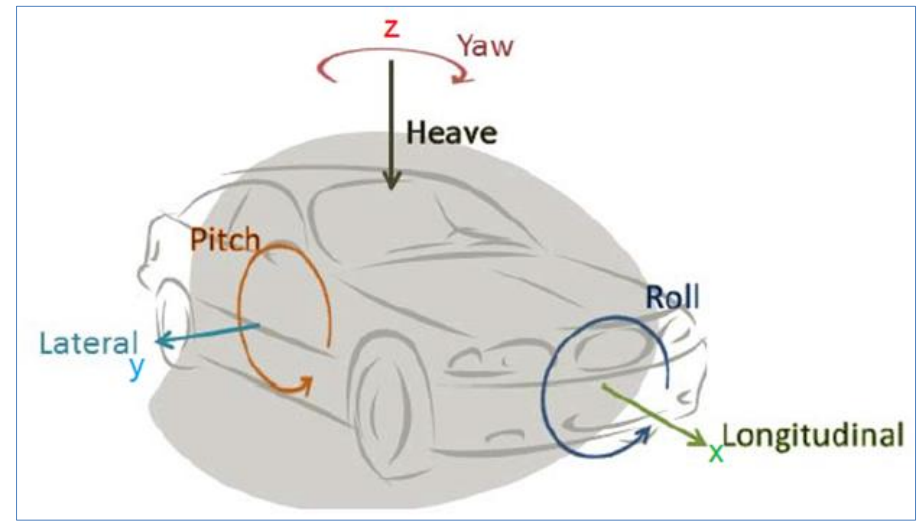

Figure 1 A system of coordinates of a vehicle fixed to COG. [10]

It is in according to the ISO standards, as described in ISO 8855. Using this coordinate system, the vehicle forward motion is depicted in the positive $\mathrm{x}$-axis and the lateral motion is depicted by the $\mathrm{y}$-axis, being positive when oriented to the driver's left side position, and the z-axis represents the vertical motion. The rotations of the vehicle cabin are also included in this system of coordinates. The pitch rotation is defined about the y-axis and the roll rotation about the $\mathrm{x}$ axis, while the yaw motion about the z-axis.

A local coordinate system will be used independently for each tire in addition to this the system of coordinates, also according to (ISO 8855). The coordinate system for a single wheel can be obtained in figure 2

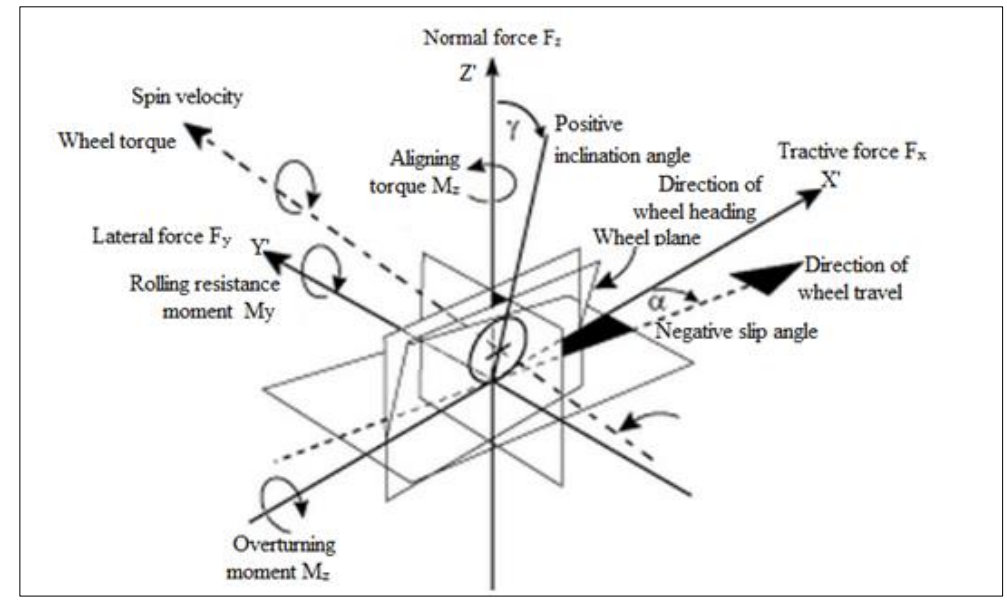

Figure 2 Wheel local coordinates system. [11] 


\section{Vehicle planar model}

The vehicle planar model is formulated from the following three equations of motion of a four-wheel vehicle with front steering. Figure 3 describes the sketch of the vehicle model and the parameters concerned. The positive $\mathrm{x}$-axis starts at the $\operatorname{cog}$ and points in the front direction of the vehicle, this direction is also indicated to as a longitudinal direction, while the $\mathrm{y}$-axis is corresponded to as the lateral direction and starts from the model centerline. It is assumed that the front wheels have the same steering angle and the roll, pitch and bob motions are neglected.

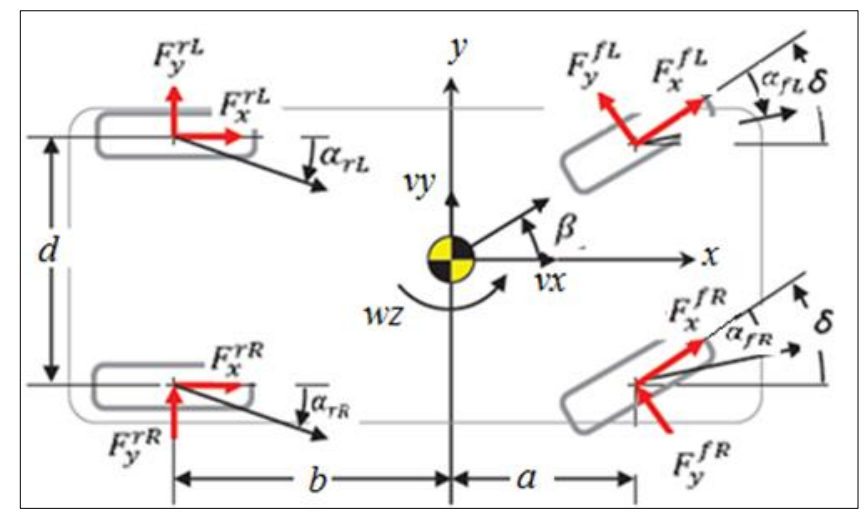

Figure 3 Vehicle planar motion model

The mathematical equations of vehicle motions can be expressed as follows:

\section{For yaw movement}

$$
\begin{gathered}
I \dot{w}_{z}=\left[a\left(F_{x}^{f R}+F_{x}^{f L}\right) \sin (\delta)+a\left(F_{y}^{f R}+F_{y}^{f L}\right) \cos (\delta)-b\left(F_{y}^{r L}+F_{y}^{r R}\right)+\right. \\
\left.\frac{d}{2}\left(F_{x}^{f R}-F_{x}^{f L}\right) \cos (\delta)+\frac{d}{2}\left(F_{x}^{r R}-F_{x}^{r L}\right)+\frac{d}{2}\left(F_{y}^{f L}-F_{y}^{f R}\right) \sin (\delta)\right]
\end{gathered}
$$

For longitudinal movement

$$
\dot{v}_{x}-v_{y} w_{z}=\frac{1}{m}\left[\left(F_{x}^{f R}+F_{x}^{f L}\right) \cos (\delta)-\left(F_{y}^{f R}+F_{y}^{f L}\right) \sin (\delta)+F_{x}^{r L}+F_{x}^{r R}\right]
$$

\section{For lateral movement}

$$
\dot{v}_{y}+v_{x} w_{z}=\frac{1}{m}\left[\left(F_{y}^{f L}+F_{y}^{f R}\right) \cos (\delta)+\left(F_{x}^{f L}+F_{x}^{f R}\right) \sin (\delta)+F_{y}^{r L}+F_{y}^{r R}\right]
$$

Whrer $F_{x}^{f L}, F_{y}^{f L} F_{x}^{f R}, F_{y}^{f R} F_{x}^{r L}, F_{y}^{r L} F_{x}^{r R}, F_{y}^{r R}$ are the components of forces for the front left tire, front right tire, rear left tire, and the rear right tire along $x$ axis and $y$ axis coordinates; $a, b$ are the displacement of the cog of the vehicle to both of front and rear axle; $l_{w}$ is the displacement between left and right tires; $v_{x}, v_{y}$ are the car longitudinal and the car lateral velocitiy, $w_{z}$ is the vehicle yaw rate, $\delta$ is the front wheel steering angle, $m$ is the vehicle total mass, $l$ is the vehicle moment inertia about its yaw.

The slip angle at each wheel ${ }^{\alpha_{i j}}$ is expressed and derived using the geometry of the vehicle and the vectors of wheel speed. If the velocity at each wheel road contact point is known then, it can easily derive the tire slip angle at each tire geometrically and can be expressed as follows: 


$$
\begin{aligned}
& \alpha_{f R}=\arctan \left(\frac{v_{y}+a \cdot w_{z}}{v_{x}+\frac{d}{2} \cdot w_{z}}\right)-\delta \\
& \alpha_{f L}=\arctan \left(\frac{v_{y}+a \cdot w_{z}}{v_{x}-\frac{d}{2} \cdot w_{z}}\right)-\delta \\
& \alpha_{r L}=\arctan \left(\frac{v_{y}-b \cdot w_{z}}{v_{x}+\frac{d}{2} \cdot w_{z}}\right) \\
& \alpha_{r L}=\arctan \left(\frac{v_{y}-b \cdot w_{z}}{v_{x}-\frac{d}{2} \cdot w_{z}}\right)
\end{aligned}
$$

The above vehicle model is analyzed and simulated using Matlab Simulink. It is assumed that the vehicle used in this case moves at a constant speed $\left(v_{x}\right)=20 \mathrm{~m} / \mathrm{s}$, the road friction coefficient is 0.4 , and the vehicle receives an input steering from the tire. Firstly, the input steering will set as a step signal, which have an amplitude of two degrees (0.035 radians) as illustrated in the figure below.

In addition, the input steering will set as a lane change maneuver with amplitude of front steering angle of 0.035 radians as obtained figure 5 .

The performance of vehicle will be obtained and compared in this thesis using the two cases of input steering angle (A step signal and a lane change maneuver).

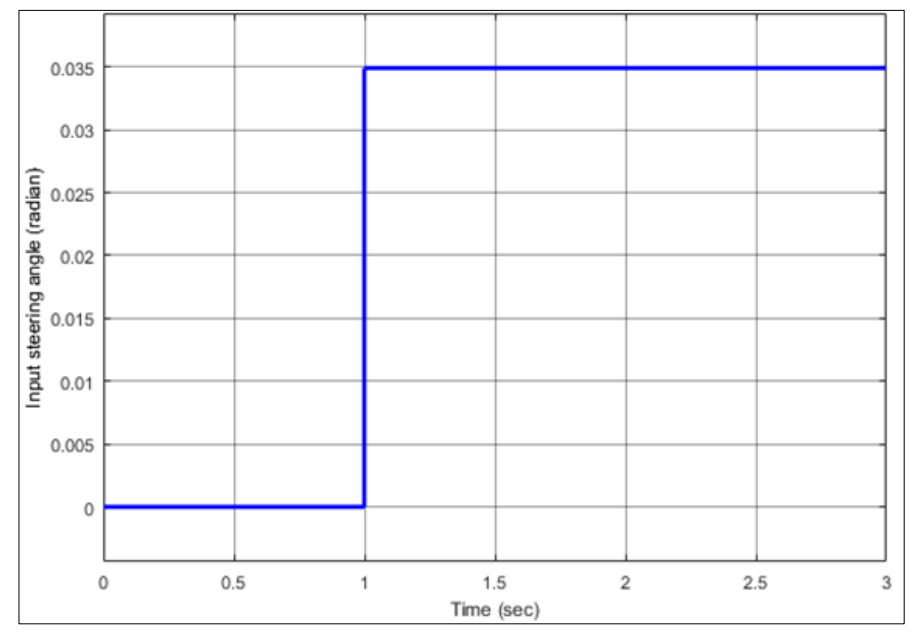

Figure 4 The steering input of vehicle maneuver

The following figures represent the performance of the car in the case of planar model that performed at a step signal of steering single and the lane change maneuver of the front wheels. The vehicle longitudinal velocity is obtained in figures 6 and 7. Figures 8 and 9 display the vehicle lateral acceleration. As it can be shown clearly, the lateral acceleration reaches its maximum rapidly during start of the second two. 


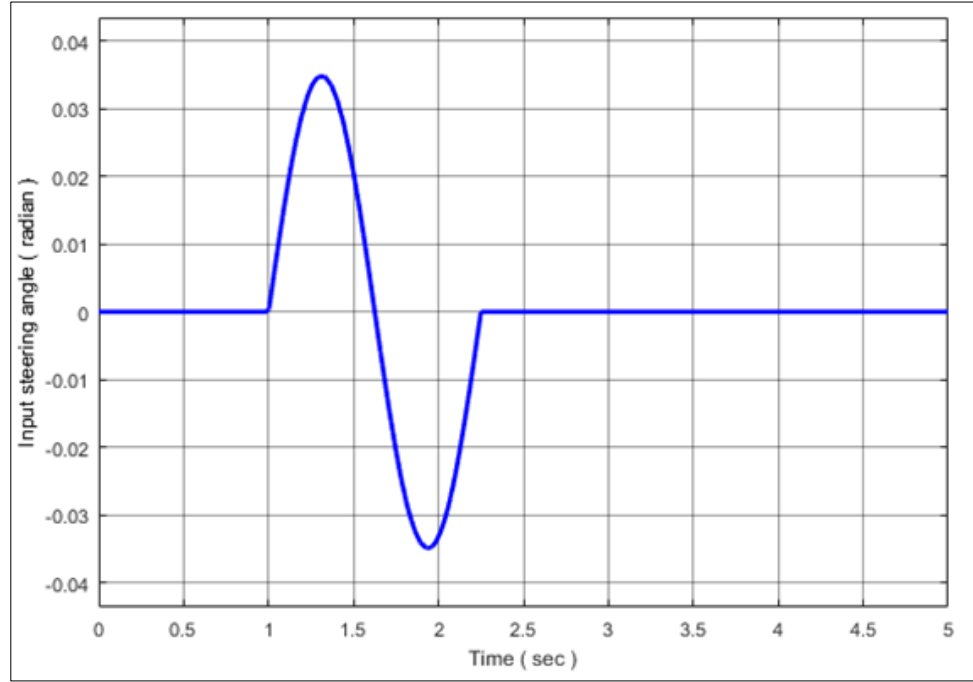

Figure 5 The steering input of vehicle with a lane change maneuver.

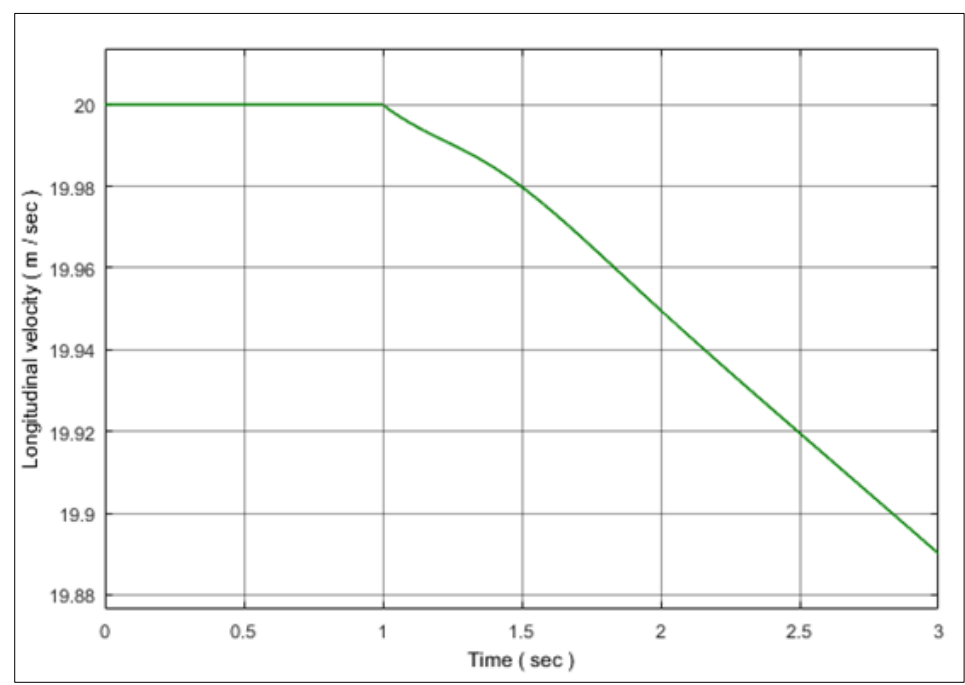

Figure 6 The vehicle longitudinal velocity at a step signal of steering angle (planar model)

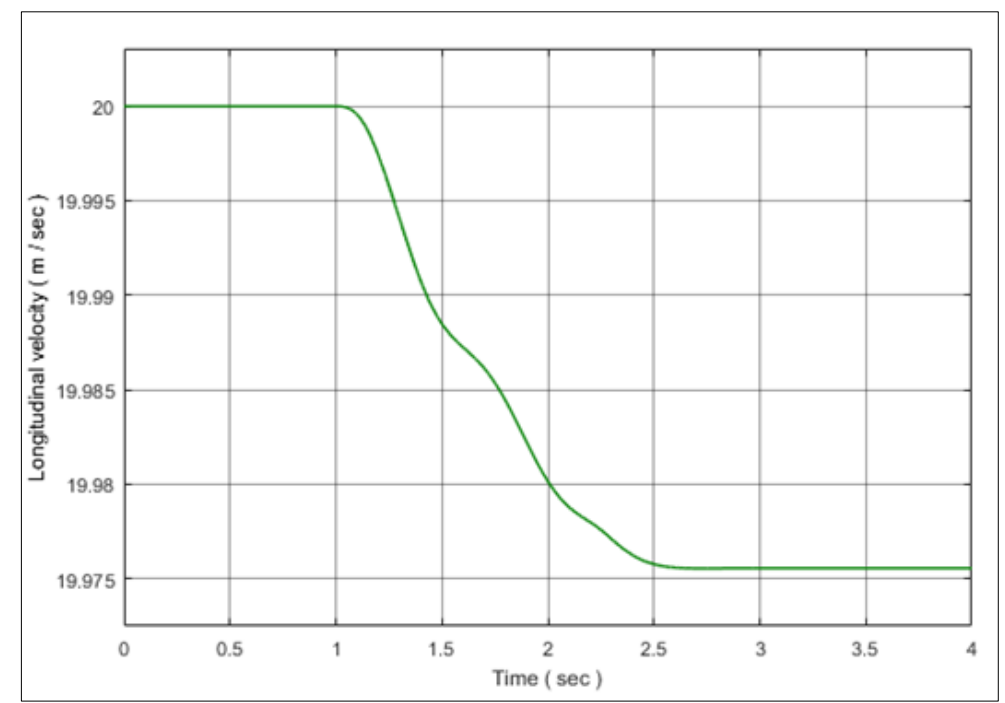

Figure 7 The vehicle longitudinal velocity on a lane change maneuver (Planar model) 
World Journal of Advanced Research and Reviews, 2021, 12(01), 331-348

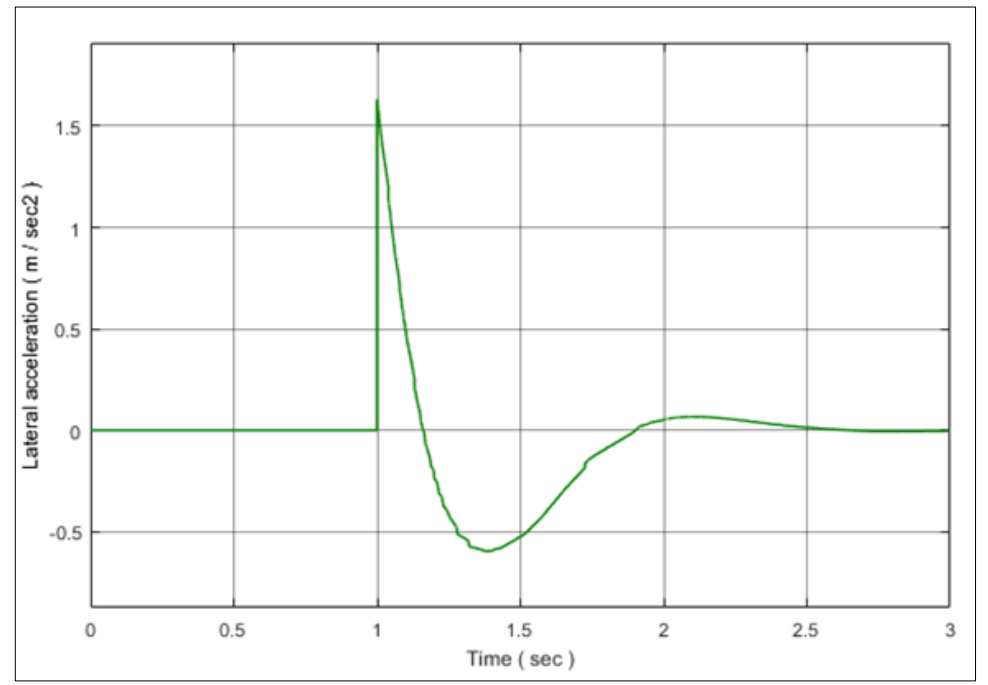

Figure 8 The vehicle lateral acceleration at a step signal of steering angle (Planar model)

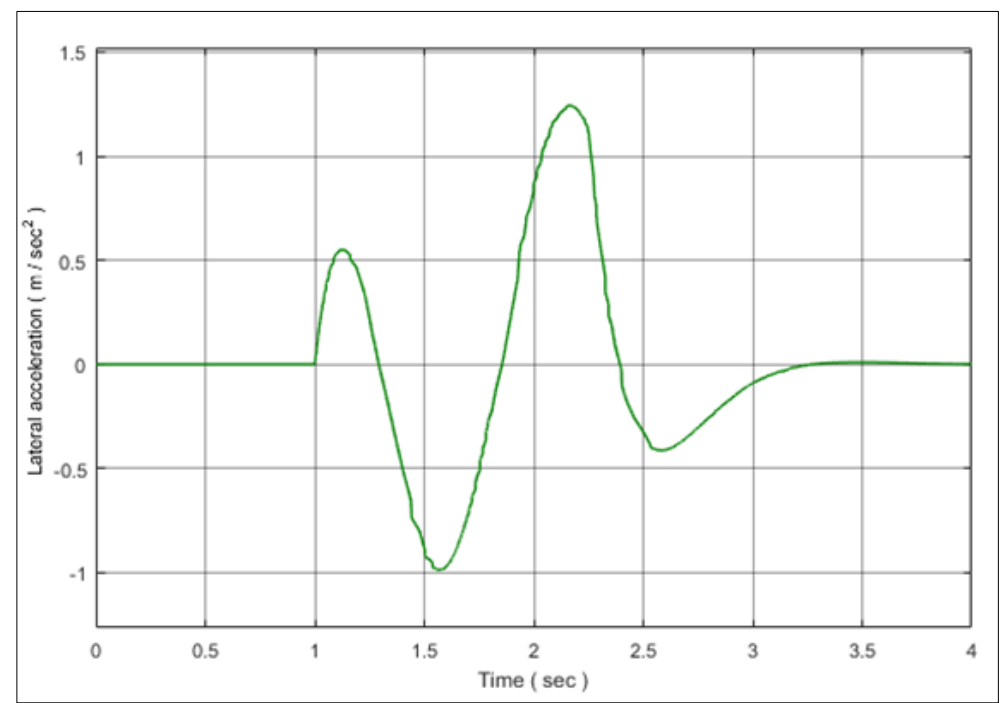

Figure 9 The vehicle lateral acceleration on a lane change maneuver (Planar model)

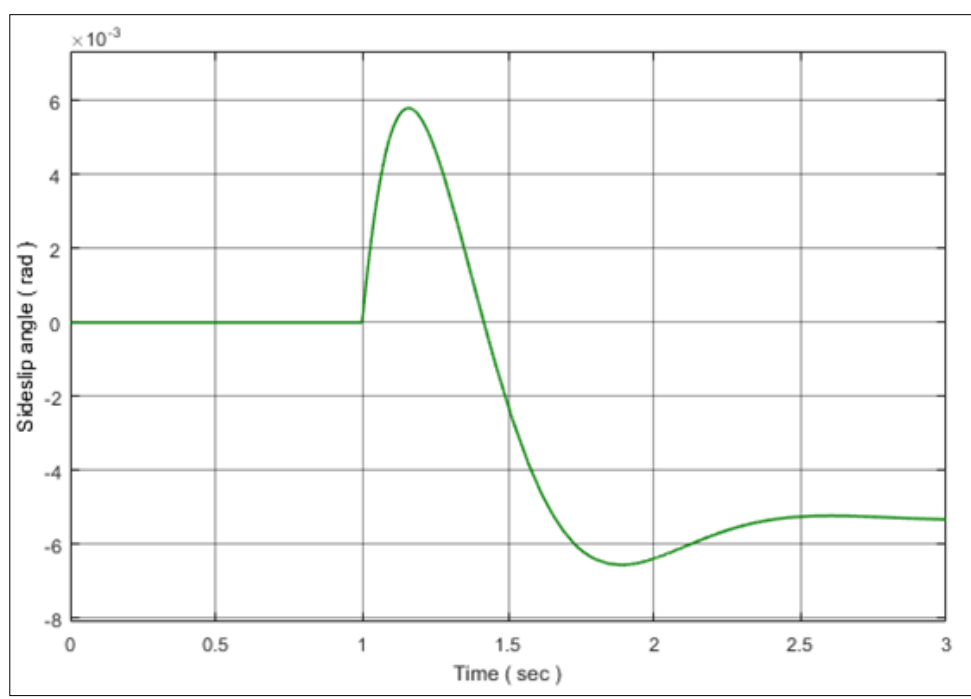

Figure 10 The vehicle sideslip angle at a step signal of steering angle (Planar model) 
World Journal of Advanced Research and Reviews, 2021, 12(01), 331-348

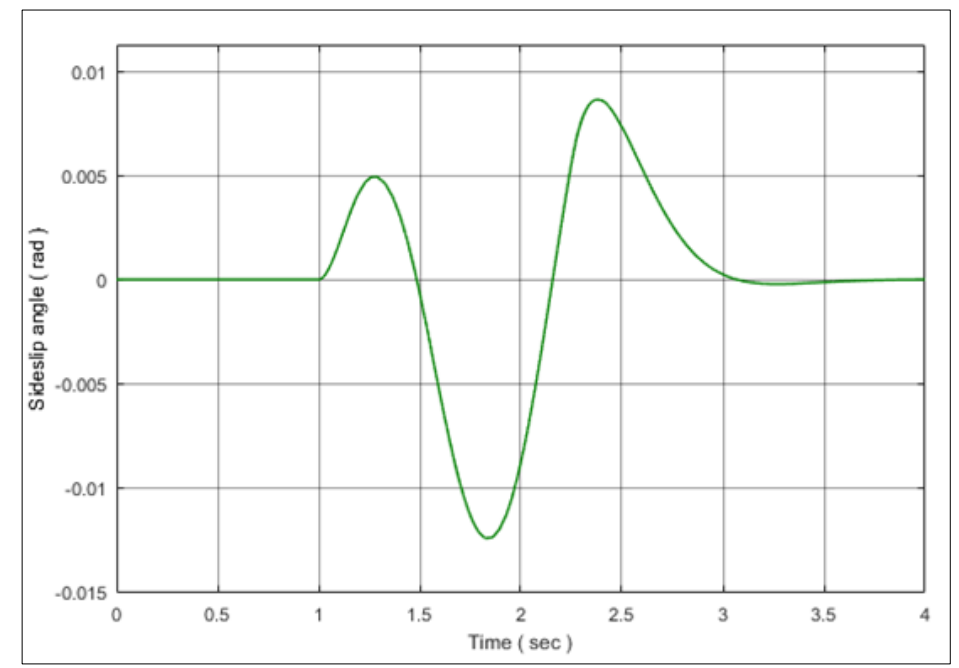

Figure 11 The vehicle sideslip angle on a lane change maneuver (Planar model)

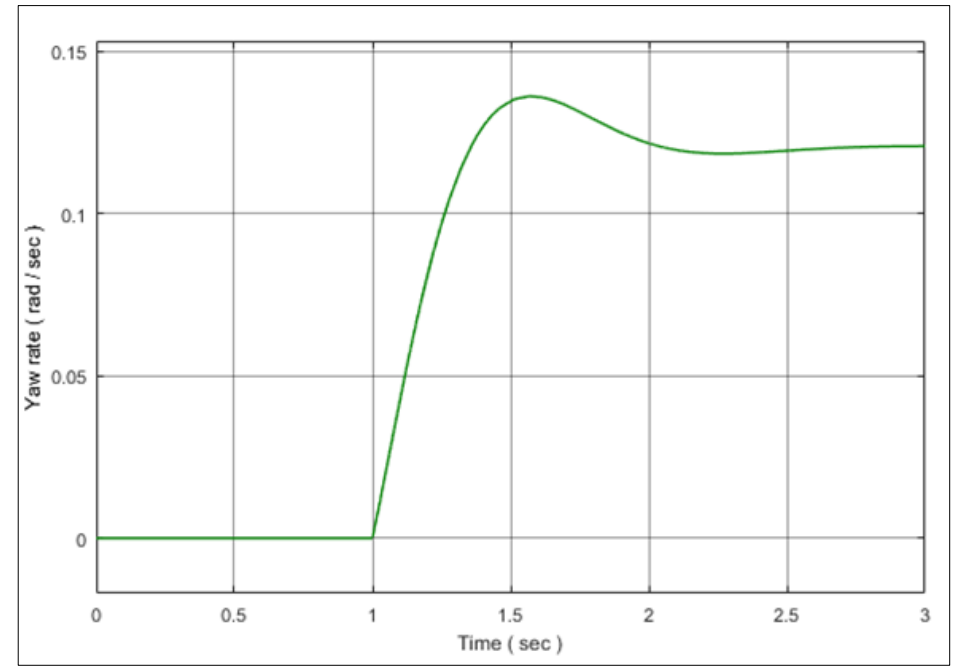

Figure 12 The vehicle yaw rate at a step signal of steering angle (Planar model)

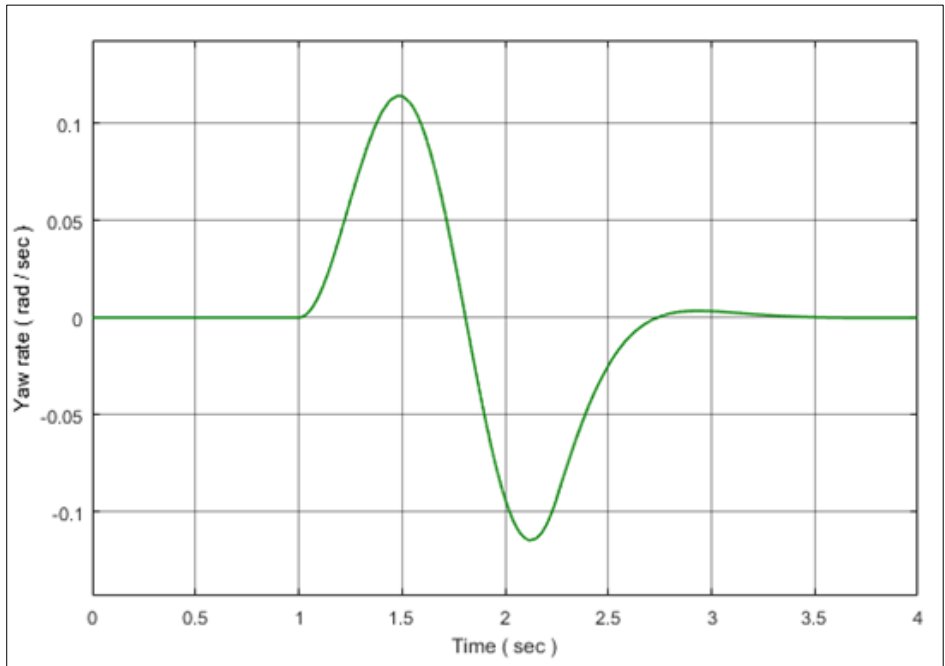

Figure 13 The vehicle yaw rate on a lane change maneuver (Planar model) 
Figures 10 and 11 show the vehicle sideslip angle change with respect to time under specified conditions and figures 12 and 13 depict the yaw rate of the vehicle versus time. It is obvious that from figures, the vehicle yaw rate at a step signal of steering angle initially increases as it is expected from the steer input. the peak value of the yaw rate at a step signal of steering angle is approximately $0.135 \mathrm{rad} / \mathrm{s}$ while the peak value of the yaw rate on a lane change maneuver is about $+0.12 \mathrm{rad} / \mathrm{s}$ and $-0.12 \mathrm{rad} / \mathrm{s}$.

\section{Full vehicle model}

In this vehicle model, the sprung mass or body is free to roll, pitch and heave, which means that the full vehicle model dynamics involves the rotation and movement on the vertical, longitudinal and lateral directions; therefore, the vehicle, vertical, longitudinal and lateral dynamics have to take into considerations for a complete car model. The suspension pats connects the body or the sprung mass to four unsprung masses that are rear-left, rear-right, front-left, and frontright tires. They are free to bouncing vertically relative to the vehicle body. The vehicle model adopted here consists of fourteen degrees of freedom (14 DOF) as obtained in the following figure.

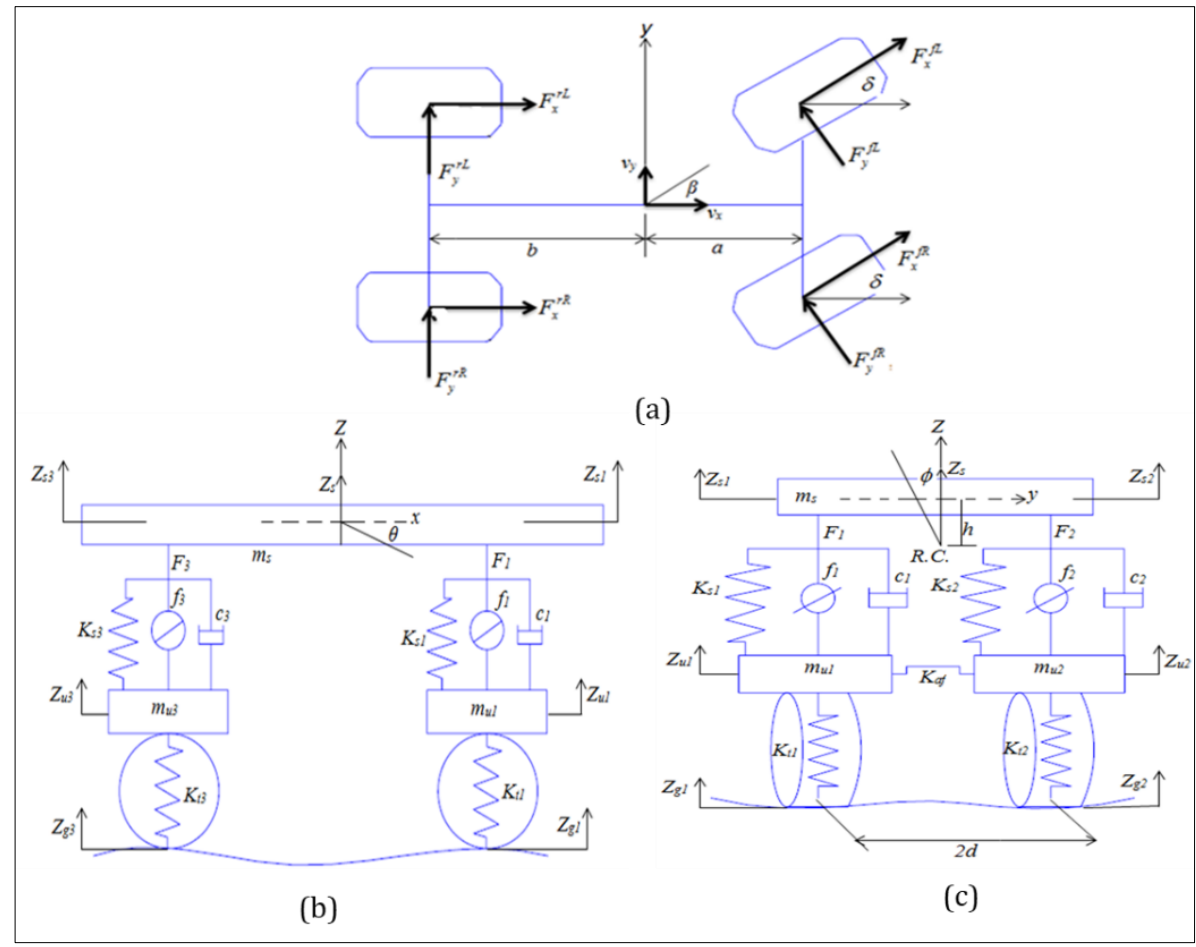

Figure 14 Full vehicle model includes: the yaw (a), pitch (b), and Roll(c) motions. [12]

The mathematical equations of the vehicle motion can be written as:

\section{For the yaw movement of the sprung mass in figure 14.a}

$$
\dot{w}_{z} I_{z}-\ddot{\phi}_{x z}=a\left(F_{x}^{f R} \sin (\delta)+F_{y}^{f R} \cos (\delta)+F_{x}^{f L} \sin (\delta)+F_{y}^{f L} \cos (\delta)\right)-b\left(F_{y}^{r L}+F_{y}^{r R}\right)
$$

Where $w_{z}$ is the vehicle yaw rate, $I_{z}$ is the sprung mass yaw moment of inertia, a and $\mathrm{b}$ is the distance between the cog. Of the vehicle and the rear, front axle, and $\varphi$ is the roll angle of sprung mass.

In addition, the differential equations of motion due to lateral and longitudinal directions can be derived as:

$$
\begin{aligned}
& m\left(\dot{v}_{x}+v_{y} w_{z}\right)-m_{s} h \dot{w}_{z} \phi=\left[F_{x}^{f R} \cos (\delta)-F_{y}^{f R} \sin (\delta)+F_{x}^{f L} \cos (\delta)\right. \\
& \left.-F_{y}^{f L} \sin (\delta)+F_{x}^{r L}+F_{x}^{r R}\right]-f_{r} m g
\end{aligned}
$$


Where $m$ is total mass of the vehicle, $v_{y}$ is the vehicle speed in the lateral direction, $v_{x}$ is the speed of the vehicle in the $\mathrm{x}$ axis direction (longitudinal direction), $f_{r}$ is the rolling resistance coefficient, and $h$ is the vertical displacement between the roll center and the cog of the sprung mass.

$$
\begin{aligned}
& m\left(\dot{v}_{y}+v_{x} w_{z}\right)+m_{s} h \ddot{\phi}=\left[\left(F_{x}^{f R} \sin (\delta)+F_{y}^{f R} \cos (\delta)+F_{x}^{f L} \sin (\delta)+\right.\right. \\
& \left.F_{y}^{f L} \cos (\delta)+F_{y}^{r L}+F_{y}^{r R}\right]
\end{aligned}
$$

The following equation describes the pitch motion of the body or sprung mass:

$$
I_{y} \ddot{\theta}=b\left(F_{z 3}+F_{z 4}\right)-a\left(F_{z 1}+F_{z 2}\right)
$$

Where $F_{z 1}, F_{z 2}, F_{z 3}, F_{z 4}$ are the total suspension forces which acting on the front and rear sprung masses, $i_{y}$ is the pitch moment of inertia of sprung mass, and $\theta$ is the sprung mass pitch angle.

In addition, for roll motion of the sprung mass:

$$
I_{x} \ddot{\phi}+m_{s}\left(\dot{v}_{y}+\dot{v}_{x} w_{z}\right) h-I_{x z} \dot{w}_{z}=m_{s} g h \phi+d\left(F_{z 2}+F_{z 3}-F_{z 1}-F_{z 4}\right)
$$

Where $i_{x}$ is the sprung mass roll moment of inertia and $I_{x z}$ is the product of inertia of the vehicle body mass about the yaw and roll axes.

The equations that describes the vertical motions of the unsprung masses sprung mass are as follows:

$$
m_{s} \ddot{Z}_{s}=F_{z 1}+F_{z 2}+F_{z 3}+F_{z 4}
$$

Where $Z_{s}$ is the vertical displacement of the vehicle body?

$$
\begin{aligned}
& m_{u 1} \ddot{Z}_{u 1}=k_{t 1}\left(Z_{g 1}-Z_{u 1}\right)-F_{z 1} \\
& m_{u 2} \ddot{Z}_{u 2}=k_{t 2}\left(Z_{g 2}-Z_{u 2}\right)-F_{z 2} \\
& m_{u 4} \ddot{Z}_{u 4}=k_{t 4}\left(Z_{g 4}-Z_{u 4}\right)-F_{z 4} \\
& m_{u 3} \ddot{Z}_{u 3}=k_{t 3}\left(Z_{g 3}-Z_{u 3}\right)-F_{z 3}
\end{aligned}
$$

$m_{u i}$ is the unsprung mass at tire $i, z_{u i}$ is the vertical distance of unsprung masses, $k_{t i}$ is the tire stiffness at tire $i$, and $z_{g} 1$ is the road excitation.

The total suspension forces which acting on the front and rear sprung masses can be expressed as:

$$
\begin{aligned}
& F_{z 1}=k_{s 1}\left(Z_{u 1}-Z_{s 1}\right)+c_{1}\left(\dot{Z}_{u 1}-\dot{Z}_{s 1}\right)-\frac{k_{a f}}{2 d}\left[\phi-\frac{\left(Z_{u 2}-Z_{u 1}\right)}{2 d}\right]+f_{1} \\
& F_{z 2}=k_{s 2}\left(Z_{u 2}-Z_{s 2}\right)+c_{2}\left(\dot{Z}_{u 2}-\dot{Z}_{s 2}\right)-\frac{k_{a f}}{2 d}\left[\phi-\frac{\left(Z_{u 2}-Z_{u 1}\right)}{2 d}\right]+f_{2} \\
& F_{z 3}=k_{s 3}\left(Z_{u 3}-Z_{s 3}\right)+c_{3}\left(\dot{Z}_{u 3}-\dot{Z}_{s 3}\right)-\frac{k_{a r}}{2 d}\left[\phi-\frac{\left(Z_{u 3}-Z_{u 4}\right)}{2 d}\right]+f_{3} \\
& F_{z 4}=k_{s 4}\left(Z_{u 4}-Z_{s 4}\right)+c_{4}\left(\dot{Z}_{u 4}-\dot{Z}_{s 4}\right)-\frac{k_{a r}}{2 d}\left[\phi-\frac{\left(Z_{u 3}-Z_{u 4}\right)}{2 d}\right]+f_{4}
\end{aligned}
$$


Where $k_{s i}$ is the suspension stiffness at each wheel, $c_{i}$ is the damper coefficient of the suspension at each wheel, $k_{a f}$ is the stiffness of anti-roll bars for the front suspension, $k_{a r}$ is the stiffness of the anti-roll bars for the rear suspension, and $f i$ is the control force of front and rear active suspension controller.

It is assumed that both of the sprung mass pitch angle $\theta$ and the sprung mass roll angle $\varphi$ have a small values, the next approximations can be achieved.

$$
\begin{aligned}
& Z_{s 1}=Z_{s}-a \theta-d \phi \\
& Z_{s 2}=Z_{s}-a \theta+d \phi \\
& Z_{s 3}=Z_{s}-b \theta+d \phi \\
& Z_{s 4}=Z_{s}+b \theta-d \phi
\end{aligned}
$$

Figures 15 to 22 represent the performance of the vehicle in the case of full model at a step signal of steering single and the lane change maneuver of the front wheels. The vehicle longitudinal velocity is obtained in figures 15 and 16, while figures 17 and 18 describe the lateral acceleration behavior of the vehicle.

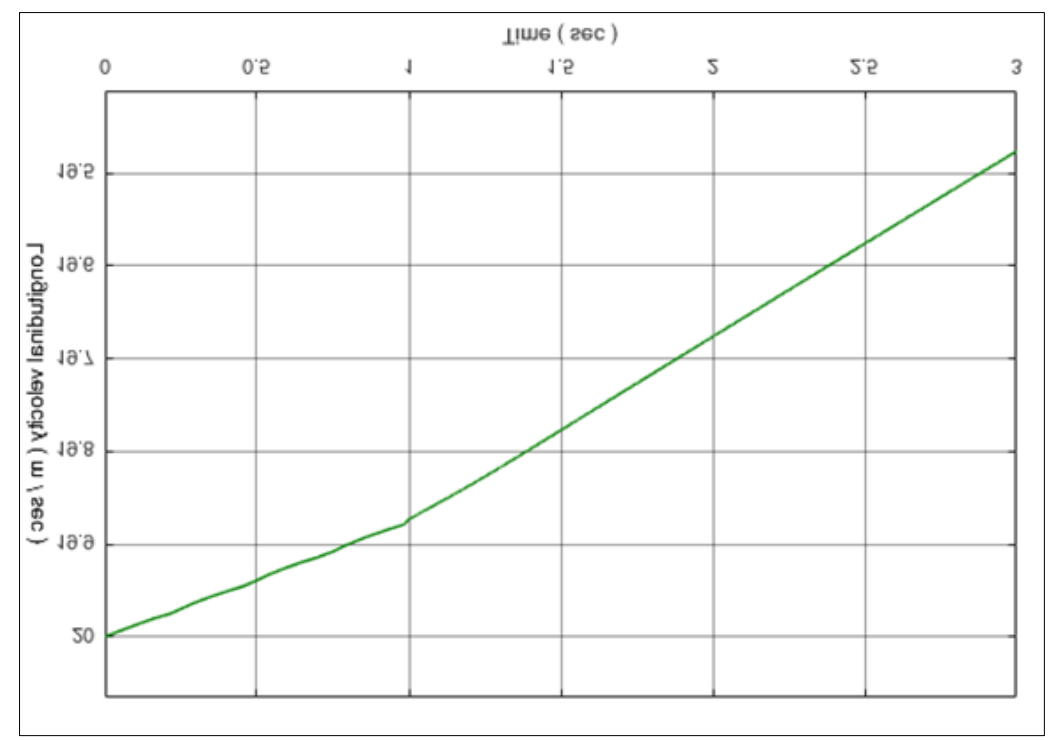

Figure 15 The vehicle longitudinal velocity at a step signal of steering angle (Full model)

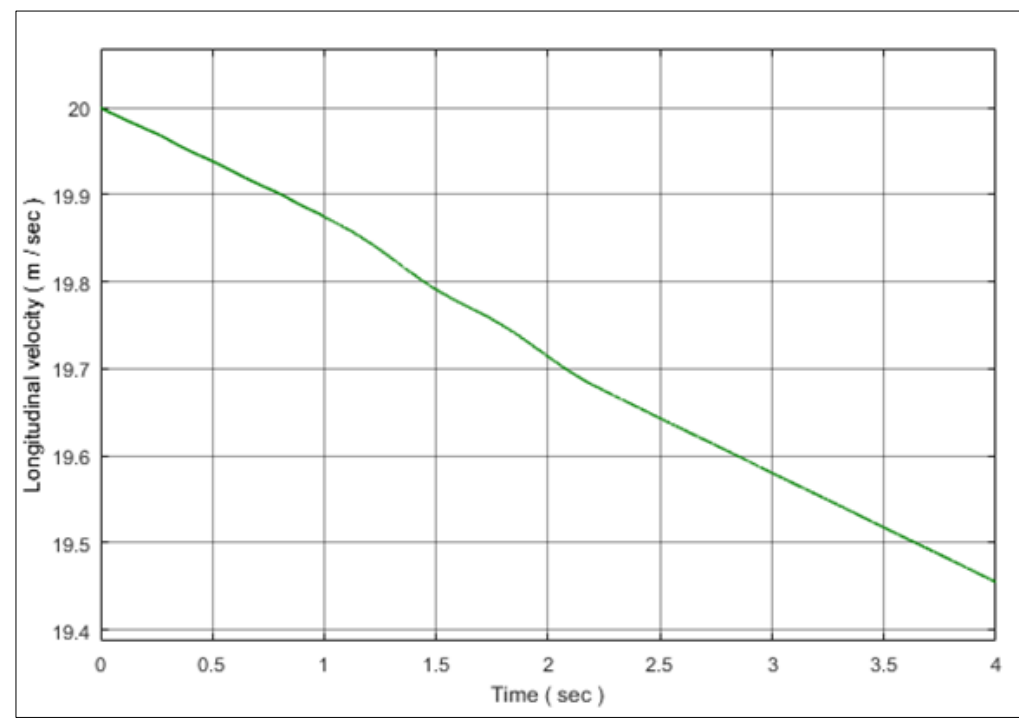

Figure 16 The vehicle longitudinal velocity on a lane change maneuver (Full model) 


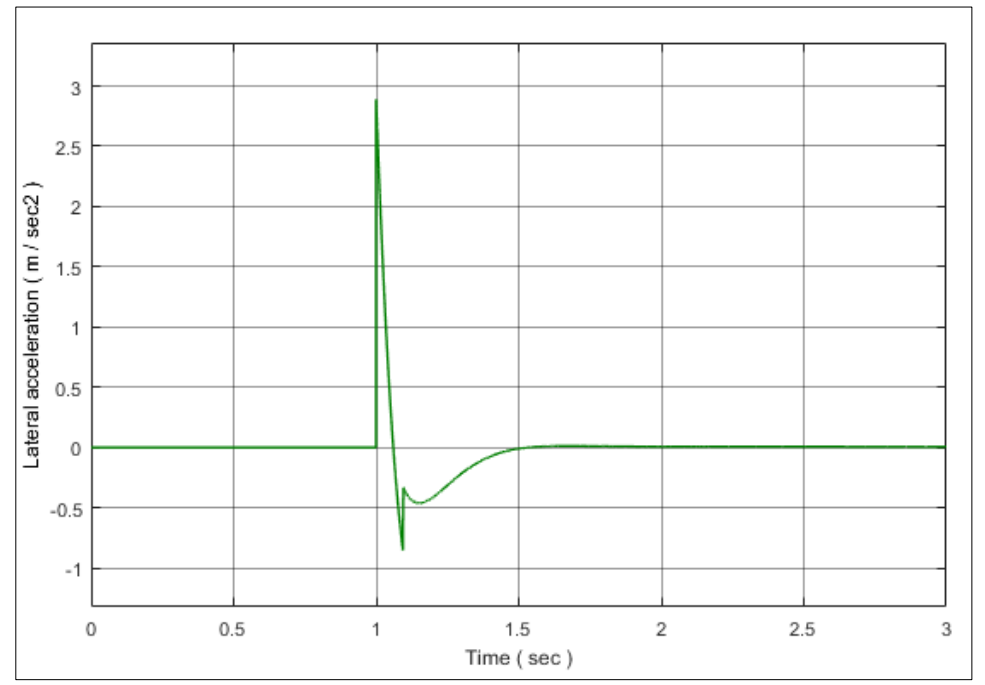

Figure 17 The vehicle lateral acceleration at a step signal of steering angle (Full model)

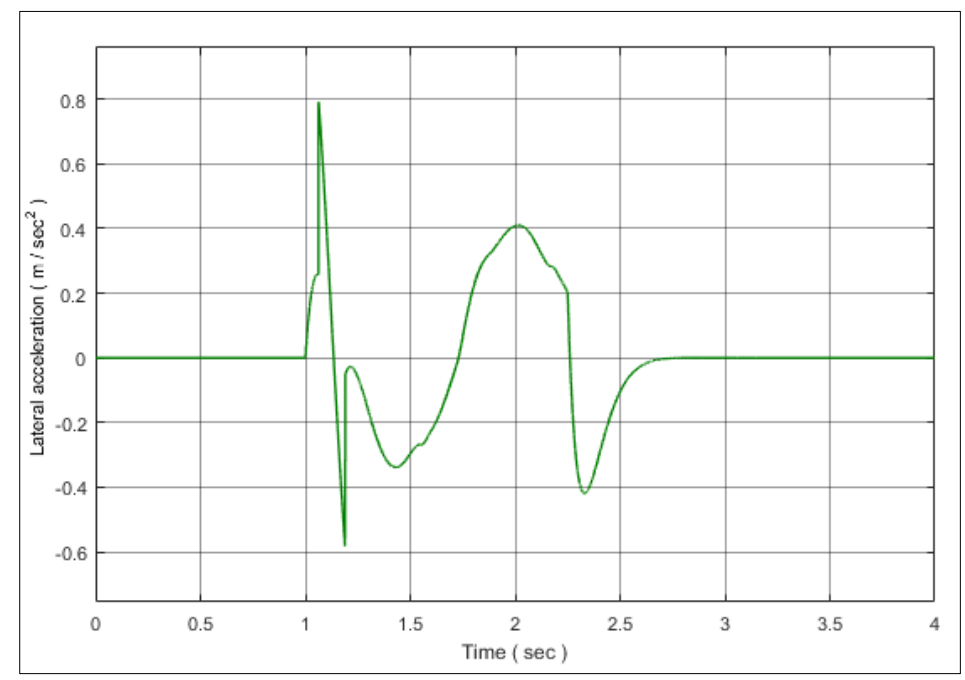

Figure 18 The vehicle lateral acceleration on a lane change maneuver (Full model)

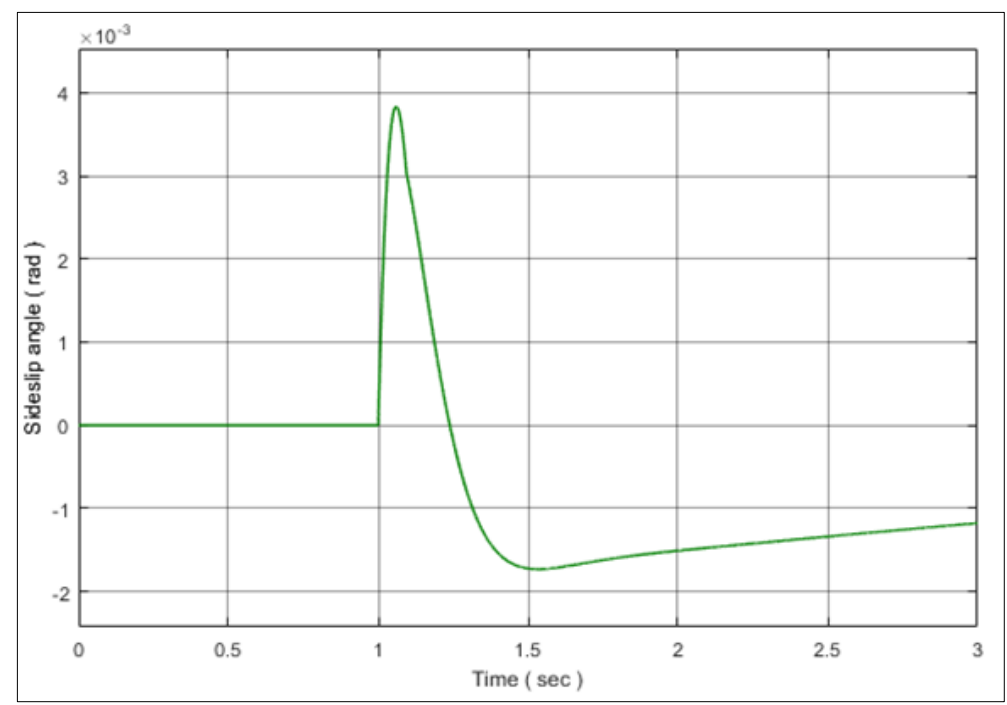

Figure 19 The vehicle sideslip angle at a step signal of steering angle (Full model) 
World Journal of Advanced Research and Reviews, 2021, 12(01), 331-348

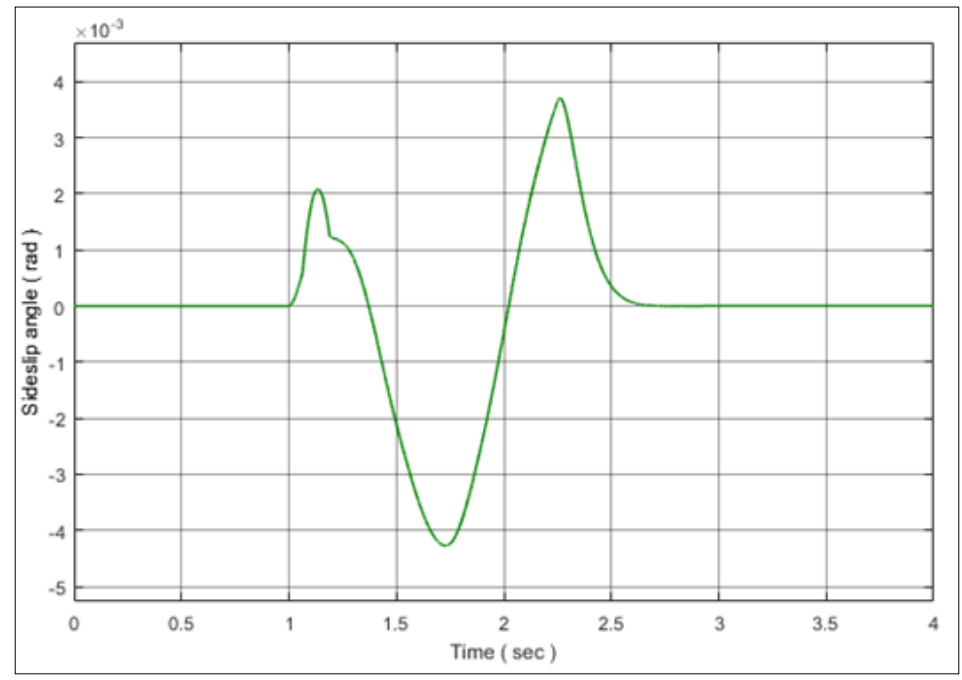

Figure 20 The vehicle sideslip angle on a lane change maneuver (Full model)

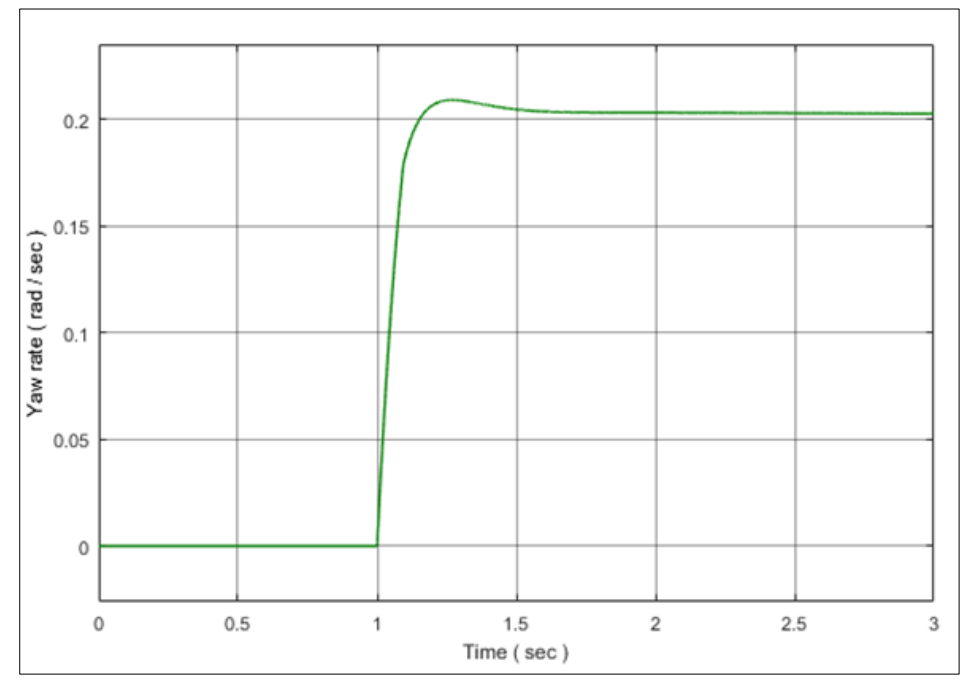

Figure 21 The vehicle yaw rate at a step signal of steering angle (Full model)

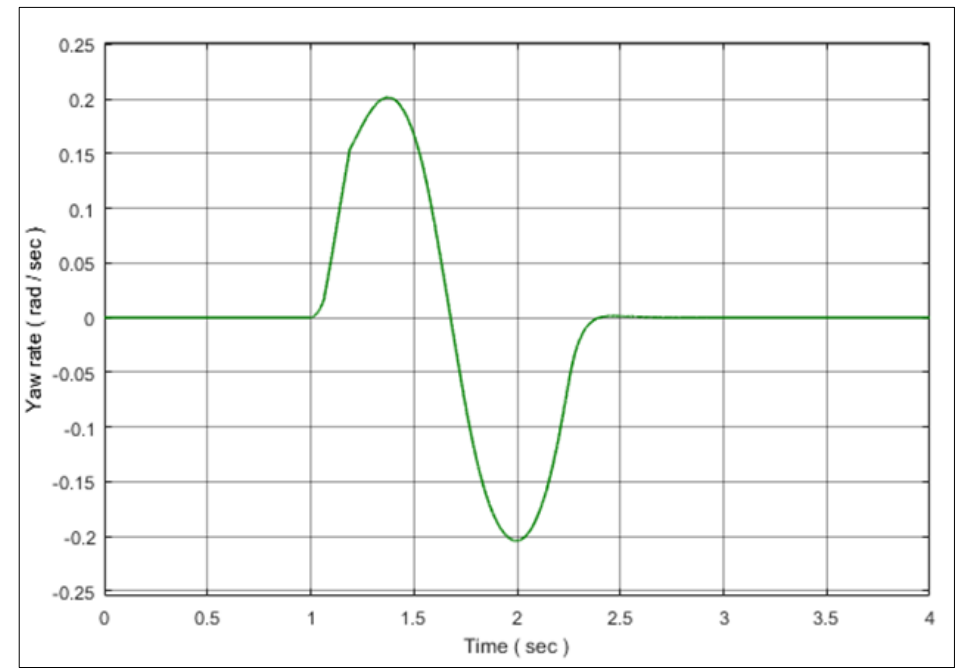

Figure 22 The vehicle yaw rate on a lane change maneuver (Full model) 
Figures 19 and 20 show the vehicle sideslip angle change with respect to time under specified conditions and figures 21 and 22 depict the vehicle yaw rate versus time. As it can be shown from the figures, the yaw rate initially increases as it is expected from the steer input. the peak value of the yaw rate at a step signal of steering angle is approximately 0.22 $\mathrm{rad} / \mathrm{s}$ while the peak value of the yaw rate on a lane change maneuver is about $+0.2 \mathrm{rad} / \mathrm{s}$ and $-0.2 \mathrm{rad} / \mathrm{s}$

\section{Vehicle reference model}

The bicycle model (reference vehicle model) as illustrated in figure 23 is an important part in the automotive engineering studies and vehicle dynamic field because it uses for controllers design and the analysis of yaw stability control prominently. according to some assumptions, it is possible to linearize the actual model of the vehicle (a nonlinear model), these assumptions are: the vehicle moves on plane surface or flat road (planar motion), the tires forces operate in a linear region, and the left and the right tires at the rear and front axle are placed in an unattached tire at the center line of a vehicle.

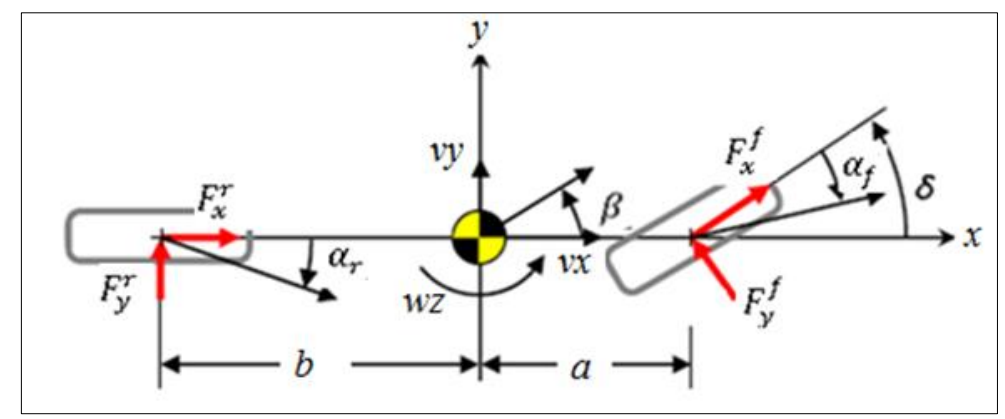

Figure 23 vehicle reference model (bicycle model)

The driver tries to control the vehicle's stability during normal and moderate cornering from the steer-ability point of view. The bicycle model gives a relationship between the stability factors and driver performance of the vehicle. Hence, the model is designed to generate desired values of the vehicle yaw rate $w_{z d}$ and the sideslip angle $\beta_{d}$ at each instance according to the driver's steering angle input and the vehicle velocity while considering of a constant forward velocity.

The following equations represent the differential equations of lateral and yaw motions of the reference model [13]:

$$
\begin{aligned}
& m v\left(\dot{\beta}_{d}-w_{z d}\right)=\left(F_{y}^{f}+F_{y}^{r}\right)-w_{z d} \\
& I_{z} w_{z d}=a \cdot F_{y}^{f}-b \cdot F_{y}^{r} \\
& F_{y}^{f}=C_{a f} \cdot \alpha_{f} \\
& F_{y}^{r}=C_{a r} \cdot \alpha_{r} \\
& \alpha_{f}=\delta-\beta_{d}-\frac{a \cdot w_{z d}}{v} \\
& \alpha_{r}=-\beta_{d}+\frac{b \cdot w_{z d}}{v}
\end{aligned}
$$

Where:

$w_{z d}, \beta_{d}$ are the desired vehicle yaw rate and desired vehicle sideslip angle.

$C_{a f}, C_{a r}$ are the longitudinal and the lateral stiffness of front wheel and rear wheel.

$a, b$ are the displacement of the cog of the vehicle to both of front and rear axle.

The simulation result for the vehicle reference model that represents the desired vehicle yaw rate is obtained in figures 24 and 25. 


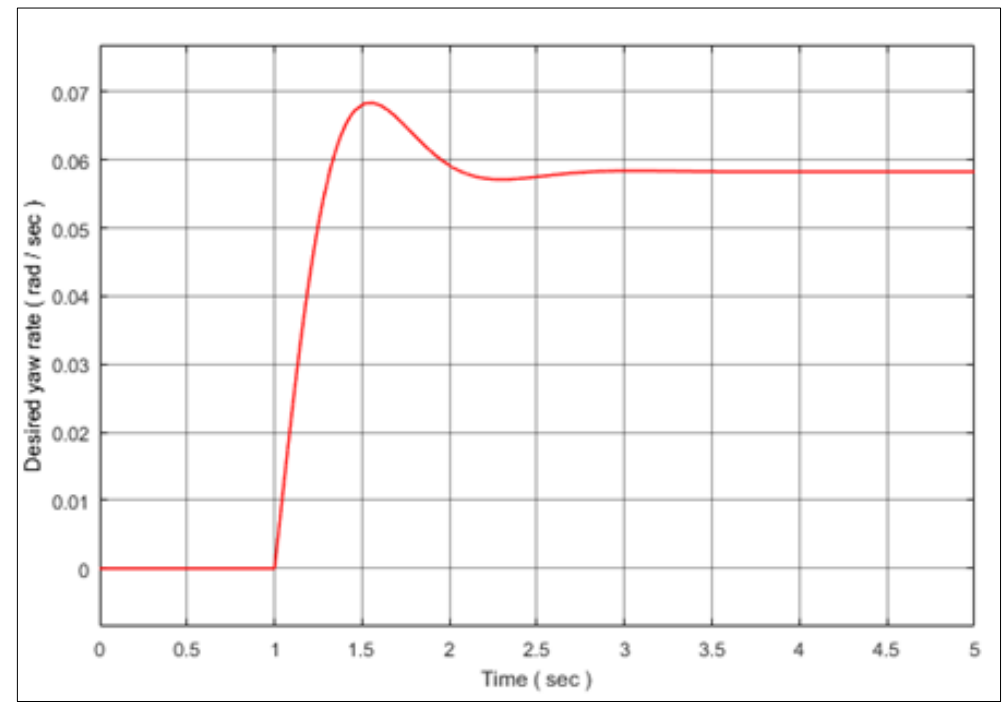

Figure 24 The desired vehicle yaw rate at a step signal of steering angle

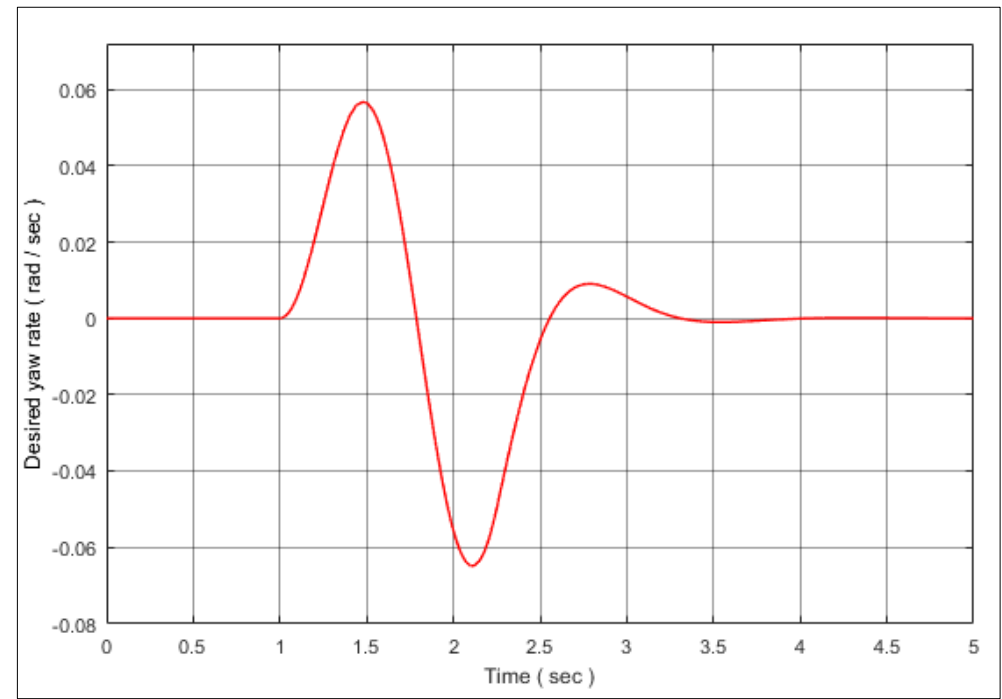

Figure 25 The desired vehicle yaw rate on a lane change maneuver

\section{Model of tire dynamic}

A sketch diagram of a modeled tire is illustrated in figure 26. The moment of inertia of the wheel is $I_{w}$ and the effective radius is $R_{w}$. The torque applied on each tire $t$ and longitudinal force of the tire $F_{x}$ is produced at the tire bottom. The tire is rotating with angular speed $\omega$ and travels at a longitudinal speed $v_{\mathrm{x}}$. The total moments about the rotation axis of the tire generates the dynamic equation derived in the following equations:

$T_{1}-F_{x 1} \cdot R_{w}=I_{w} \dot{\omega}_{1}$

$T_{2}-F_{x 2} \cdot R_{w}=I_{w} \dot{\omega}_{2}$

$T_{3}-F_{x 3} \cdot R_{w}=I_{w} \dot{\omega}_{3}$

$T_{4}-F_{x 4} \cdot R_{w}=I_{w} \dot{\omega}_{4}$ 


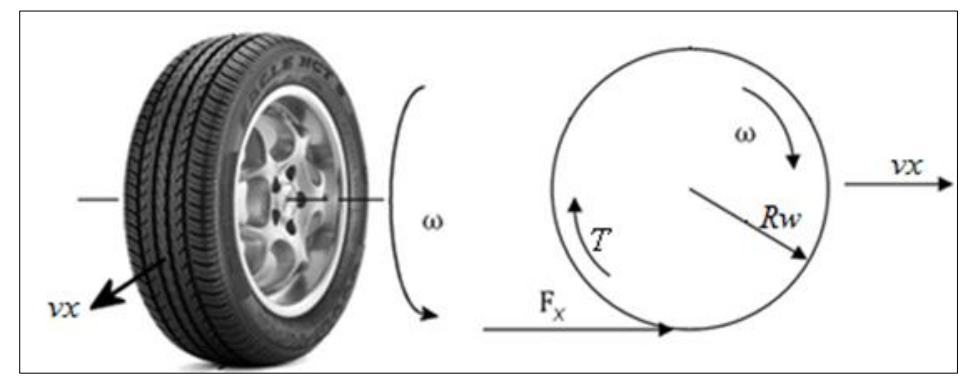

Figure 26 Wheel schematic diagram

\section{Review of existing tire models}

The widely used experiential tire model is called the magic tire formula that can be used to fit empirical tire data for describing the relationship between the longitudinal force of the tire and slip ratio, the lateral tire force and the slip angle, and the relationship between self-aligning moment and the slip angle. It is derived as follows:

$$
\begin{aligned}
& y(x) D \sin \{C \arctan [B x-E(B x-\arctan B x)]\} \\
& Y(X)=y(x)+S_{v} \\
& x=X+S_{h}
\end{aligned}
$$

Where $y(x)$ indicates the output variables: the longitudinal and lateral tire forces, or self- align moment, and $\mathrm{x}$ indicates slip angle $\alpha$ or slip ratio $\sigma$. The coefficient B is the stiffness factor, $\mathrm{C}$ is called shape factor, $\mathrm{D}$ is peak factor, and $\mathrm{E}$ the factor of curvature. $S_{v}$ and $S_{h}$ are called the vertical shift and horizontal shift, respectively.

The curve which crosses through the origin point, $x=y=0$ is produced from last equation, and reaches the maximum where $X=\mathrm{Xxm}$, as illustrated in figure 3.27 for the given values coefficients, the curve presents an antisymmetric style with respect to the origin point, $x=y=0$. Figure 27 shows the physical meaning of the coefficients mentioned above.). For example, if figure 27 represents the relationship between the slip angle and lateral tire force, then coefficient D indicates the higher value with respect to $x$ and $y$ coordinates and the factor BCD represents to the curve slop in the origin point, representing the tire cornering stiffness.

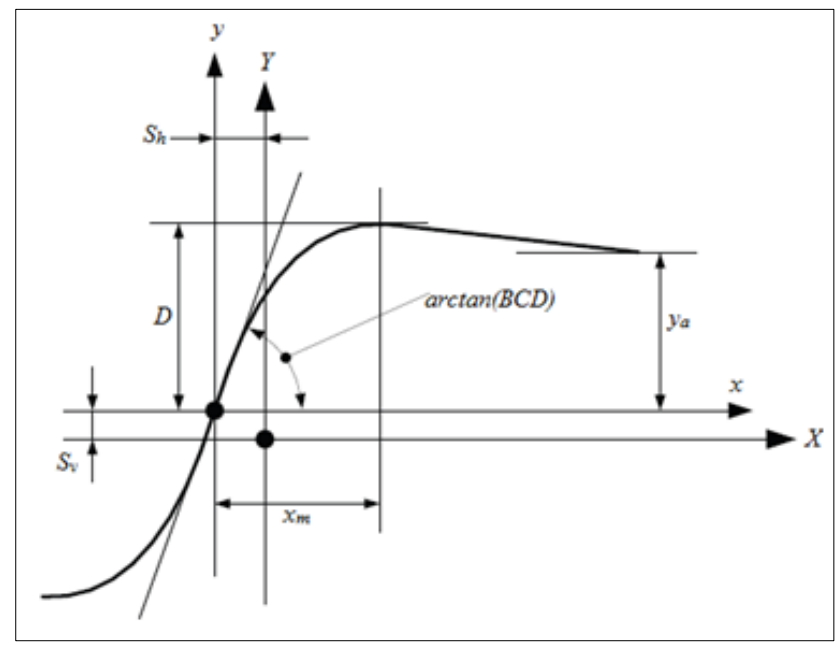

Figure 27 Characteristics of the magic formula. [14]

It is noted that some of the coefficients in the last two equations are a functions of the vertical tire force $\mathrm{F}^{\mathrm{z}}$ and/or camber angle of tire. Coefficients B, C, D, and C can be derived as functions of the vertical tire force $F^{z}$ and the road friction coefficient $\mu$ as follows:

$$
D=a_{1}\left(F^{z}\right)^{2}+a_{2} F^{z}
$$

Where $a_{1}$ and $a_{2}$ are experiential coefficients. 
For stiffness of tire cornering (i.e., the slope of the lateral tire force and slip angle curve):

$$
(B C D)=a_{3} \sin \left[a_{4} \arctan \left(a_{5} F^{z}\right)\right]
$$

And for the align stiffness (the slope of the self-aligning moment and the slip angle curve) or the longitudinal stiffness (the slope of the longitudinal tire force and the slip ratio):

$$
B C D=\frac{a_{3}\left(F^{z}\right)^{2}+a_{4} F^{z}}{e^{a_{5} F^{z}}}
$$

The stiffness factor $\mathrm{b}$ and the curvature factor e are given by:

$$
\begin{gathered}
B=\frac{B C D}{C D} \\
E=a_{6}\left(F^{z}\right)^{2}+a_{7} F^{z}+a_{8}
\end{gathered}
$$

Where $a_{1}, a_{2}, \cdots, a_{8}$ are empirical coefficients.

Another widely used of tire models is the Dugoff's tire formula. The Dugoff's model represents an interesting alternate to the elastic foundation of analytical tire model modified by Fiala in 1954 for the generation of lateral tire force and by sharp and Pacejka for combined lateral and longitudinal and forces generation. The Dugoff model permits independent tire stiffness values in both of the lateral and longitudinal directions. Compared to magic formula, the Dugoff tire model has the merit of being an analytical derived formula developed from the calculations of force balance. Furthermore, the lateral and longitudinal forces of the tire have a direct relation to the road-tire friction coefficient in more clear equations [14].

The longitudinal $F_{x}$ and lateral $F_{y}$ tire forces are defined as follows:

$$
\begin{aligned}
& F_{x}=C_{1} \frac{\sigma_{x}}{1+\sigma_{x}} f(\lambda) \\
& F_{y}=C_{2} \frac{\tan \alpha}{1+\sigma_{x}} f(\lambda)
\end{aligned}
$$

Where $C_{1}$ and $C_{2}$ are the longitudinal and cornering tire stiffness, ${ }_{x}$ is the tire longitudinal slip ratio and can be defined in last two equations, and $\alpha_{i}$ is the tire slip angle at each tire.

$$
\begin{gathered}
\sigma_{x}=1-\frac{r_{e f f} \omega_{i}}{v_{x} \quad \text { In the case of deceleration }} \\
\sigma_{x}=-1+\frac{v_{x}}{r_{e f f} \omega_{i}} \text { In the case of acceleration }
\end{gathered}
$$

The slip angle for each tire is derived and calculated depend on the geometry dimensions of the vehicle and the wheel speed vectors. If it is possible to determine the velocity at each wheel road contact point then, it can derive the tire slip angle easily at each tire geometrically and can be expressed as follows:

$$
\begin{aligned}
& \alpha_{f R}=\arctan \left(\frac{u+L_{f} \cdot r}{v+L_{w} \cdot r}\right)-\delta \\
& \alpha_{f L}=\arctan \left(\frac{u+L_{f} \cdot r}{v-L_{w} \cdot r}\right)-\delta
\end{aligned}
$$




$$
\begin{gathered}
\alpha_{r L}=\arctan \left(\frac{u-L_{r} \cdot r}{v+L_{w} \cdot r}\right) \alpha_{r L}=\arctan \left(\frac{u-L_{r} \cdot r}{v-L_{w} \cdot r}\right) \\
\text { The variable } \lambda \text { and the function } f(\lambda) \text { are given by } \\
\lambda=\frac{\mu F_{z}\left(1+\sigma_{x}\right)}{2 \sqrt{\left\{\left(C_{1} \sigma_{x}\right)^{2}+\left(C_{2} \tan (\alpha)\right)^{2}\right\}}}
\end{gathered}
$$

And

$$
\begin{aligned}
& f(\lambda)=(2-\lambda) \lambda \quad \text { if } \lambda<1 \\
& f(\lambda)=1 \quad \text { if } \lambda \geq 1
\end{aligned}
$$

Where $\left({ }^{\mu}\right)$ is the coefficient of road-tire friction and $F_{i}{ }^{z}$ represents the vertical tire force for each wheel.

\section{Conclusion}

This paper showed a set of important mathematical models needed by everyone interested in studying the dynamics and control of vehicles such as the vehicle planar model, full vehicle model, and the two degrees of freedom vehicle model (bicycle model), in addition to clarifying the main parameters when studying the performance of cars such as the vehicle longitudinal velocity, the vehicle lateral acceleration, the vehicle sideslip angle, and the vehicle yaw rate. It is recommended in future work to present and evaluate the control methods used when studying the performance of vehicles and comparing them.

\section{Compliance with ethical standards}

\section{Disclosure of conflict of interest}

The authors: Mohamed Belrzaeg, Abdussalam Ali Ahmed, Amhimmid Q Almabrouk, Mohamed Mohamed Khaleel, Alforjani Ali Ahmed and Meshaal Almukhtar declare that they have no conflict of interest.

\section{References}

[1] Bengt Jacobson et al, Vehicle Dynamics Compendium, Chalmers University of Technology, Bengt Jacobson. 2017.

[2] Manfred MMM. Henning Wallentowitz. Vehicle system dynamics. Tsinghua University Press, Beijing. 2009.

[3] Dave C, Yu F. Vehicle system dynamics and control. China Communications Press, Beijing. 2004.

[4] Segel L. An overview of developments in road vehicle dynamics: past, present and future. In: Proceedings of IMechE conference on vehicle ride and handling, London. 1993; 1-12.

[5] Abe M. Vehicle handling dynamics: theory and application. Butterworth Heinemann, Oxford. 2009.

[6] Guo KH. Vehicle handling dynamics theory. Jiangsu Science and Technology Press, Nanjing. 2011.

[7] Rajesh R. Vehicle dynamics and control. Springer-Verlag, New York. 2005.

[8] Zhang LJ, He H. Vehicle dynamics theory and application. National Defense Industry Press, Beijing. 2011.

[9] Yu ZS. Automotive theory, 5th edn. China Machine Press, Beijing V. 2010.

[10] Rana Raouf Hasan Farag"Active Neuro-Fuzzy Integrated Vehicle Dynamics Controller To Improve The Vehicle Handling And Stability At Complicated Maneuvers" Phd Thesis. July 2013.

[11] Jorge Gómez Fernández "A Vehicle Dynamics Model For Driving Simulators" Master Thesis, Chalmers University. 2012.

[12] Wuwei Chen, Hansong Xiao, Liqiang Liu, Jean W. Zu and Huihui Zhou," Integrated Control Of Vehicle System Dynamics: Theory and Experiment, Advances in Mechatronics", Intech. 2011. 
[13] M. K. Aripin, Yahaya Md Sam, Kumeresan A. Danapalasingam, Kemao Peng, N. Hamzah, Andm. F. Ismail," A Review of Active Yaw Control System for Vehicle Handling and Stability Enhancement", International Journal of Vehicular Technology. 2014; 15.

[14] R Rajamani, Vehicle Dynamics and Control: Springer Science \& Business Media. 2011.

[15] Rana Raouf Hasan Farag"Active Neuro-Fuzzy Integrated Vehicle Dynamics Controller To Improve The Vehicle Handling And Stability At Complicated Maneuvers" Phd Thesis. July 2013.

[16] L Feiqiang, W Jun, L Zhaodu. "On The Vehicle Stability Control For Electric Vehicle Based On Control Allocation," In 2008 Ieee Vehicle Power And Propulsion Conference. 2008; 1-6.

[17] H Xiao, W Chen, H Zhou, JW Zu, "Integrated Vehicle Dynamics Control Through Coordinating Electronic Stability Program And Active Suspension System," In 2009 International Conference On Mechatronics And Automation. 2009; 1150-1155.

[18] Yin Guodong, Jin Xianjian, Qing Zhiyong \& Bian Chentong," Lateral Stability Region Conservativeness Estimation And Torque Distribution For Fwia Electric Vehicle Steering",Science China, Technological Sciences. April 2015; 58 (4): 669-676.

[19] YE Ko, CK Song." Vehicle Modeling With Nonlinear Tires For Vehicle Stability Analysis",International Journal Of Automotive Technology. 2010; 11(3): 339-344.

[20] Kangwon Lee, Youngwoo Kim And Jinhee Jang," A Study Of Integrated Chassis Control Algorithm With Brake Control And Suspension Control Systems For Vehicle Stability And Handling Performance",Icros-Sice International Joint Conference, August 18-21, 2009, Japan.

[21] Jorge Gómez Fernández "A Vehicle Dynamics Model For Driving Simulators" Master Thesis, Chalmers University. 2012.

[22] AA Ahmed, AF Saleh Alshandoli, "Using Of Neural Network Controller And Fuzzy PID Control To Improve Electric Vehicle Stability Based On A14-DOF Model," 2020 International Conference on Electrical Engineering (ICEE). 2020; 1-6.

[23] D Fodor, K Enisz, R Doman, P Toth. "Tire road friction coefficient estimation methods comparison based on different vehicle dynamics models," 2011 IEEE Vehicle Power and Propulsion Conference. 2011; 1-4.

[24] H Guo, Z Yin, D Cao, H Chen, C Lv. "A Review of Estimation for Vehicle Tire-Road Interactions Toward Automated Driving," in IEEE Transactions on Systems, Man, and Cybernetics: Systems. Jan. 2019; 49(1): 14-30.

[25] Duan-Feng Chu, Gang-Yan Li, X. Lu and J. K. Hedrick, "A combined dynamics model for active roll stability control of ground vehicles," 2010 2nd International Conference on Computer Engineering and Technology. 2010; V5303-V5-307.

[26] G Tagne, R Talj, A Charara. "Design of passivity-based controllers for lateral dynamics of intelligent vehicles," 2015 IEEE Intelligent Vehicles Symposium (IV). 2015; 1044-1049.

[27] SM Fard, A Khajepour, A Goodarzi, E Esmailzadeh. "Design and control of a narrow electric vehicle," 2012 IEEE International Electric Vehicle Conference. 2012; 1-7.

[28] K Jiang, A Victorino, A Charara, "Estimation and prediction of vehicle dynamics states based on fusion of OpenStreetMap and vehicle dynamics models," 2016 IEEE Intelligent Vehicles Symposium (IV). 2016; $208-213$.

[29] Qunzhi Zhou and Feiyue Wang, "Driver assisted fuzzy control of yaw dynamics for 4WD vehicles," IEEE Intelligent Vehicles Symposium. 2004; 425-430.

[30] Abdussalam Ali Ahmed, vehicle stability optimization based on fourteen degree of freedom model and using of neural network controller, Euro Asia 8th. International congress on applied sciences Tashkent, Uzbekistan. March 2021; 15-16.

[31] S Kulothungan, RV Anirudh, K Sivashankar, AK Dash. "Design and Development of a Vehicle Dynamics Model for a Drive Simulator," 2018 3rd International Conference on Communication and Electronics Systems (ICCES). 2018; 153-156.

[32] AA Ahmed and OSM Jomah. "Vehicle Yaw Rate Control For Lane Change Maneuver Using Fuzzy PID Controller And Neural Network Controller," 2020 IEEE 2nd International Conference on Electronics, Control, Optimization and Computer Science (ICECOCS). 2020; 1-6. 